\section{O custo da extensão da vigência de patentes de medicamentos para o Sistema Único de Saúde}

\author{
The cost to the Brazilian Unified National Health \\ System of the extension of drug patent terms
}

\author{
El coste para el Sistema Único de Salud de Brasil \\ de la extensión de la vigencia de patentes de \\ medicamentos
}

Julia Paranhos 1

Eduardo Mercadante 1

Lia Hasenclever 1,2

\section{Resumo}

O parágrafo único do art. 40 da Lei de Propriedade Industrial brasileira estabelece a extensão de vigência de patentes pela mora do exame, ao menos, até dez anos a partir da concessão. Pensado como exceção, esse dispositivo tornouse a regra no caso das patentes farmacêuticas, prolongando o prazo de exclusividade de exploração de medicamentos. Isso impacta o consumo privado e o orçamento do Sistema Único de Saúde (SUS). Este trabalho investiga o custo, para o SUS, da extensão de vigência de nove medicamentos, por ser proibida a compra de versões genéricas ou biossimilares. Foram analisados três grupos: (a) quatro já em extensão; (b) três que, com a concessão das patentes, terão extensão; e (c) dois com patentes mailbox que estão em domínio público, mas que podem ter a extensão garantida judicialmente. A partir de valores unitários e quantidades totais de compra pelo Departamento de Logística em Saúde do Ministério da Saúde (DLOG), foi estimado um gasto total de R\$ 10,6 bilhões com os nove medicamentos entre 2014 a 2018. Assim, foi projetado o gasto total nos respectivos períodos de extensão: $R \$ 6,8$ bilhões. Uma busca de mercado por preços de alternativas genéricas retornou uma redução potencial de $R \$ 1,2$ bilhão para o gasto do DLOG com apenas quatro medicamentos. Por fim, com base nas reduções mínimas, médias e máximas do mercado, foi encontrada uma economia potencial de até $R \$ 3,9$ bilhões no gasto do DLOG com a compra dos nove medicamentos, se não houvesse o dispositivo de extensão de vigência.

Patente; Preço de Medicamento; Custos e Análise de Custo; Sistema Único de Saúde

\author{
Correspondência \\ J. Paranhos \\ Instituto de Economia, Universidade Federal do Rio de Janeiro. \\ Av. Pasteur 250, sala 130, Rio de Janeiro, RJ 22290-902, Brasil. \\ juliaparanhos@ie.ufrj.br \\ ${ }^{1}$ Instituto de Economia, Universidade Federal do Rio de Janeiro, \\ Rio de Janeiro, Brasil. \\ 2 Universidade Candido Mendes, Campos dos Goytacazes, \\ Brasil.
}




\section{Introdução}

A dinâmica de geração da inovação na indústria farmacêutica é fortemente ligada ao sistema de propriedade industrial. O longo tempo de pesquisa e desenvolvimento (P\&D) de um produto, os altos riscos envolvidos na probabilidade de sucesso desse investimento e a necessidade cada vez maior de fontes externas de conhecimento fazem com que os direitos de propriedade industrial formais tenham grande importância no retorno dos investimentos, reforçando a estratégia de apropriabilidade sobre as inovações farmacêuticas $1,2,3,4$. No entanto, o uso de tais mecanismos cria barreiras à entrada de concorrentes, o que gera poder de mercado aos detentores e definição de preços a níveis elevados 5,6,7. Isso gera significativos efeitos sobre os orçamentos de saúde, públicos ou privados.

O tema da propriedade intelectual ganha relevância no caso brasileiro, posto que o país: (a) tem o maior sistema público de saúde do mundo, o Sistema Único de Saúde (SUS); (b) apresenta grande dependência tecnológica externa na área farmacêutica; (c) antecipou a harmonização do seu sistema de propriedade industrial ao Acordo TRIPS (Trade-Related Aspects of Intellectual Property Rights Agreement), assinado em dezembro de 1994, com a implementação da Lei de Propriedade Industrial (LPI) - Lei no 9.279, em 1996; e (d) incluiu na legislação elementos que vão além dos parâmetros mínimos obrigatórios do Acordo, normalmente chamados de TRIPS-plus ou TRIPS-extra 8,9,10,11.

O SUS foi criado em 1988 com base na universalidade da saúde prevista no art. 196 da Constituição Federal. Em 2018, o orçamento do SUS foi da ordem de R\$121,86 bilhões, tendo sido executado 88,77\% desse valor: R\$108,18 bilhões (Governo Federal. Portal da Transparência. http://www.portal transparencia.gov.br, acessado em 22/Abr/2019). Desde 2013, o percentual do orçamento gasto com medicamentos tem ficado acima de $13 \% 12$. Isso faz com que as compras públicas de medicamentos, nas três esferas de governo, representem cerca $20 \%$ do mercado farmacêutico brasileiro 13 . Dado que a compra pública de medicamentos conta com recursos limitados, qualquer impeditivo ou atraso na ampliação da concorrência gera efeitos negativos sobre a sustentabilidade do SUS e dificuldade de acesso a medicamentos pela população.

Por sua vez, a LPI foi promulgada em 1996 e entrou em vigor em sua integralidade em 199714. Essa foi uma decisão TRIPS-plus, porque o Acordo exigia que o Brasil estivesse harmonizado apenas a partir de 2005, por ser um país de renda média e por ter que reintroduzir o patenteamento farmacêutico, excluído desde 1945 para produtos e 1969 para processos 8,9. Portanto, o Brasil teve um período de transição de apenas dois anos e meio, abdicando dos dez anos permitidos pelo TRIPS. Durante esse período de transição, somente podiam ser depositados pedidos de patente mailbox ou pipeline ${ }^{8,11}$. O mecanismo mailbox, definido em TRIPS, permitia aos pedidos que passariam a ser patenteáveis após a harmonização que fossem depositados - para garantir a novidade e titularidade - e aguardassem a transição para então serem examinados. $\mathrm{O}$ mecanismo pipeline permitia que patentes farmacêuticas já concedidas anteriormente fora do país fossem depositadas no Brasil e concedidas apenas com base em exame formal, e não técnico, desde que não tivessem sido comercializadas em nenhum lugar do mundo, nem houvesse esforços significativos para a exploração de seus objetos no Brasil.

Dessa maneira, o mecanismo mailbox já conferia alguma proteção aos inventores farmacêuticos no período de transição, tornando o instrumento pipeline um elemento TRIPS-extra, porque não tem qualquer embasamento no Acordo, além de ir contra seus próprios parâmetros, ao permitir proteção sem novidade - posto que as patentes já haviam sido concedidas em outros países 11. Essas mudanças conferiram um significativo aumento no nível de proteção intelectual no país a partir de meados da década de 1990, interrompendo de forma prematura o processo de produção local de medicamentos 8,15 .

O não aproveitamento do período de transição também foi prejudicial ao Escritório de Patentes brasileiro, o Instituto Nacional da Propriedade Industrial (INPI). Por ter sido criado em 1970, não possuía experiência no exame de patentes farmacêuticas. Portanto, no momento de promulgação da LPI, foi preciso iniciar a construção da área com contratação e formação de avaliadores ${ }^{8}$. Alguns dispositivos da LPI buscaram lidar com a expectativa de dificuldade administrativa na implementação do patenteamento farmacêutico. Por um lado, há a determinação no art. 229-C de que a Agência Nacional de Vigilância Sanitária (Anvisa) deveria emitir um parecer pela anuência da concessão de patentes farmacêuticas. Esse instrumento foi criado para aproveitar o pessoal técnico da Anvisa qualificado para o exame de patentes farmacêuticas 9,16. Por outro, o parágrafo único do art. 40 14, incluído 
na legislação para servir de exceção nos casos em que o tempo de análise fosse muito longo, é um elemento TRIPS-plus 10,11, porque apenas seu caput era exigência do Acordo (grifo nosso):

"Art. 40. A patente de invenção vigorará pelo prazo de 20 (vinte) anos e a de modelo de utilidade pelo prazo 15 (quinze) anos contados da data de depósito.

Parágrafo único. O prazo de vigência não será inferior a 10 (dez) anos para a patente de invenção e a 7 (sete) anos para a patente de modelo de utilidade, a contar da data de concessão, ressalvada a hipótese de o INPI estar impedido de proceder ao exame de mérito do pedido, por pendência judicial comprovada ou por motivo de força maior"

No entanto, mesmo com os artifícios criados para solucionar a falta de estrutura do INPI, suas condições de funcionamento ainda ficam aquém do nível dos grandes escritórios internacionais. $\mathrm{O}$ levantamento de Mercadante 11 sobre dados de 2017 permite colocar o INPI em perspectiva internacional, comparando-se com a Índia, o México e os cinco maiores escritórios (IP5): Estados Unidos, China, Japão, Coreia do Sul e o Escritório Europeu. Desses oito, o Brasil é o que tem o quarto maior backlog, apesar de ser o sétimo em número de depósitos no ano. Combinando esse grande volume de pedidos pendentes de decisão final com o baixo número de examinadores de patente ( $323 \mathrm{em}$ janeiro de 2019) - só tem mais que o México -, o país termina com a pior razão de backlog por examinador. Em janeiro de 2019, essa razão alcançou 641 pedidos por examinador 17.

A morosidade no exame de patentes do INPI é especial no setor farmacêutico. As patentes farmacêuticas levam, em média, 13 anos para receber decisão final de concessão, indeferimento ou arquivamento técnico, sendo o campo tecnológico de maior lentidão 18 . Essa média elevada tem uma consequência direta no tempo de vigência das patentes, pois torna o parágrafo único do art. 40 uma regra e não uma exceção, como inicialmente previsto: 92,2\% das patentes que podem ter extensão, e que já foram concedidas, terão vigência superior a 20 anos 11 .

Para um país com forte dependência tecnológica e um grande sistema público de saúde, esse fato tem efeitos perversos sobre o aumento dos custos de aquisição de medicamentos, visto que adia a entrada de concorrentes no mercado. A regulação do setor determina que somente após o fim da proteção patentária podem ser produzidas cópias dos medicamentos de referência: genéricos e similares para os de síntese química, e biossimilares para os biológicos.

Pelo menos dois estudos já publicados, mas utilizando metodologias distintas, identificaram os efeitos perversos para o SUS da extensão de patentes pelo parágrafo único do art. 40. No primeiro estudo, realizado em 2016, a análise potencial de custo adicional para o SUS, relativo a nove medicamentos com patentes com prazo estendido, era de $R \$ 2,1$ bilhões 19 . No segundo estudo 20 , indicou-se um custo adicional potencial de $\mathrm{R} \$ 288,4$ milhões para três medicamentos antirretrovirais.

Considerando-se a permanência do parágrafo único do art. 40 e o fato de o seu uso tornar-se a regra, é relevante realizar novas análises dos efeitos para o SUS dos custos adicionais de medicamentos com patentes com extensão. Portanto, este artigo realiza uma pesquisa com metodologia original, com foco em análises potenciais do prejuízo para o SUS da extensão da vigência de patentes de medicamentos já estendidas ou na eminência de virem a ser, o que adiaria a abertura desses mercados específicos para a competição. O objetivo geral deste artigo consiste em fazer uma nova e atualizada projeção dos custos adicionais para o SUS da compra de medicamentos que tiveram ampliação do seu período de exclusividade pela extensão da vigência da patente, devido ao parágrafo único do art. 40. Os objetivos específicos são: (a) determinar uma amostra representativa das compras públicas de medicamentos protegidos por patente, entre 2014 e 2018, cujas vigências foram ou podem ser estendidas; (b) identificar os gastos atuais do Ministério da Saúde com a compra desses medicamentos; (c) identificar o período de extensão de vigência das patentes que protegem os medicamentos comprados; (d) estimar os gastos com a compra pelo Ministério da Saúde dos medicamentos nos respectivos períodos de extensão de vigência; e (e) calcular estimativas do custo potencial por ano e total para o Ministério da Saúde, considerando que poderia haver disponibilidade de genéricos ou biossimilares para compra, a partir de 20 anos da data de depósito da patente, se não houvesse extensão. 


\section{Metodologia}

Esta pesquisa consiste em um estudo de caso descritivo e prospectivo da compra pública de medicamentos com proteção patentária estendida no Brasil. A pergunta de pesquisa que direcionou o estudo foi: "Qual é o custo adicional potencial para o SUS da extensão das patentes de medicamentos pelo parágrafo único do artigo 40 da Lei de Propriedade Industrial?”.

O cálculo dessa estimativa foi realizado em oito etapas de pesquisa. Na primeira etapa, os gastos com compras de medicamento do Ministério da Saúde foram analisados, restringindo o levantamento às compras do Departamento de Logística em Saúde, da Secretaria Executiva (DLOG). As informações sobre as compras do DLOG foram extraídas em 18 de fevereiro de 2019 do Painel de Compras do Governo (http://paineldecompras.economia.gov.br/), para o período de 2014 a 2018, considerando as compras efetivamente realizadas por dispensa de licitação e inexigibilidade de licitação.

Na segunda etapa, foram selecionados os medicamentos e as respectivas patentes para realização dos cálculos de custo potencial. Foram destacados os 50 medicamentos que representaram maiores gastos para o DLOG de 2014 a 2018. Todos os valores foram atualizados para janeiro de 2019 com base no Índice de Preços ao Consumidor Amplo (IPCA) específico para saúde e cuidados pessoais, calculado pelo Instituto Brasileiro de Geografia e Estatística (IBGE). Nessa lista, foram encontrados nove medicamentos de alto custo para o SUS com patentes com extensão ou com potencial de extensão.

Em seguida, foram criados três grupos representativos dos medicamentos protegidos por patentes. Foram agrupadas todas as concentrações do mesmo medicamento. No entanto, não foram agrupadas as diferentes composições. Por exemplo, a combinação de sofosbuvir e ledipasvir não foi considerada. O Grupo A representa os quatro medicamentos cujas patentes já foram concedidas com extensão: golimumabe 50mg, da Janssen-Cilag; daclatasvir 30mg e 60mg, da Bristol-Myers Squibb; dasatinibe 20mg, 50mg e 100mg, da Bristol-Myers Squibb; e nilotinibe 200mg, da Novartis. O Grupo B engloba os três medicamentos cujos pedidos de patente ainda não receberam a decisão final do INPI, porém foram depositados há mais de 10 anos da data de coleta das informações: certolizumabe pegol 200mg, da AstraZeneca; darunavir 75mg, 150mg e 600mg, da Janssen-Cilag; e sofosbuvir 400mg, da Gilead. O Grupo C comporta os dois medicamentos cujas patentes já foram concedidas, porém são pedidos mailbox, cuja extensão de vigência é questionada judicialmente: adalimumabe $40 \mathrm{mg}$, da AbbVie; e eculizumabe $10 \mathrm{mg} / \mathrm{mL}$, da Alexion.

A inclusão do Grupo C se justifica pela tentativa dos titulares de conseguirem judicialmente a extensão, mesmo que a LPI expressamente afirme que patentes mailbox não possuem essa garantia (art. 229, parágrafo único). É necessário destacar que o DLOG continuou comprando os dois medicamentos de referência do Grupo C, sem substituição por biossimilares, mesmo após o fim de sua vigência. Não obstante as considerações sobre os desafios tecnológicos da produção de biossimilares, foi adotada neste artigo a tese de que a insegurança jurídica causada pelo processo judicial atrasa a entrada de concorrentes no mercado, tal qual uma expectativa de extensão, análoga à expectativa de direito do art. 44 da LPI. Esse dispositivo assegura que o depositante de um pedido de patente tem a expectativa de adquirir o direito exclusivo sobre a invenção após a concessão da patente; logo, uma vez concedida, ele pode exigir reparação retroativa por eventuais infrações que tenham sido cometidas enquanto o pedido ainda estava tramitando no INPI. Dessa maneira, o presente artigo analisa casos efetivos de extensão (Grupo A), casos potenciais dentro do marco regulatório vigente (Grupo B) e casos potenciais se houver mudança na previsão legal (Grupo C).

$\mathrm{Na}$ terceira etapa, foram calculados indicadores de valor, quantidade e gasto para cada medicamento. O valor médio unitário (VMU) foi estimado a partir da média dos valores unitários de cada compra, ponderada pelas respectivas quantidades e pelas diferentes concentrações, quando houver. A quantidade média anual (QMA) foi obtida a partir da divisão do total de unidades compradas pelo número de anos de incorporação ao SUS, ponderando as diferentes concentrações. Especificamente para o Grupo C, como as patentes estão extintas enquanto seus titulares tentam conseguir a extensão, foram calculados o VMU e QMA apenas para os períodos em que os medicamentos não estavam legalmente protegidos pela patente, não obstante a expectativa de extensão. Foram considerados todos os gastos efetivos desde 10 de fevereiro de 2017 para o adalimumabe, e desde 1o de maio de 2015 para o eculizumabe. A partir de 2019, foram estimados os gastos potenciais, considerando que o adalimu- 
mabe ainda teria 1,1 ano de extensão, e o eculizumabe, 0,8 ano. Dessa maneira, foi possível calcular o gasto médio anual (GMA) para todos os medicamentos.

Na quarta etapa, foram identificadas as informações das patentes que protegem cada medicamento: números das patentes e dos pedidos de patente relativos aos medicamentos selecionados, bem como suas datas de depósito e, quando possível, suas datas de concessão. Como apresentado no Quadro 1, apenas foi considerada uma patente para cada medicamento, significando que este artigo não aborda a extensão do tempo de proteção efetiva por meio de estratégias evergreening, que consistem em cercar os objetos de proteção de diversas patentes, ampliando ainda mais o potencial de onerar o SUS. O único fato gerador de extensão aqui considerado foi a morosidade do exame dos pedidos e a consequente garantia do prazo mínimo de vigência prevista no parágrafo único do art. 40 da LPI.

$\mathrm{Na}$ quinta etapa, foi calculado o tempo de extensão da proteção de cada medicamento a partir das datas indicadas no Quadro 1. É importante notar que a extensão do Grupo A é efetiva, porque foi concedida após mais de dez anos de exame. A extensão do Grupo B é potencial, pois se assume que foi concedida em 18 de fevereiro de 2019, data de coleta dos dados deste artigo. A extensão do Grupo C é também potencial, dado que, apesar de as patentes terem sido concedidas sem extensão, a sua solicitação de extensão encontra-se em análise no judiciário.

Na sexta etapa, foram levantados os preços unitários de medicamentos genéricos ou biossimilares disponíveis no mercado internacional que poderiam ser vendidos ao DLOG no caso da não extensão da patente dos medicamentos estudados. Foram encontrados valores de genéricos mais baratos apenas para quatro medicamentos, todos de base química. Três preços unitários dos genéricos correspondem a compras públicas em países de renda baixa ou média: daclatasvir $60 \mathrm{mg}$ por US\$ 0,25; darunavir $600 \mathrm{mg}$ por US\$ 0,90; e sofosbuvir $400 \mathrm{mg}$ por US\$ 0,79 21,22,23. O quarto genérico teve seu preço obtido diretamente do site da empresa produtora: dasatinibe 50mg, por US\$ 4,28 (CAPRANE. Dasa-50: dasatinibe. https://www.caprane.com/product/dasa-50-dasatinib-50mg/, acessado em 22/ Abr/2019). Apesar de ser um preço de varejo, é menor que o pago pelo DLOG. Também foram

\section{Quadro 1}

Patentes que protegem os medicamentos.

\begin{tabular}{|c|c|c|c|c|c|}
\hline Grupo/Medicamento & Patente & Depósito & Concessão * & Extinção ** & Extensão (anos) \\
\hline \multicolumn{6}{|l|}{ A } \\
\hline Golimumabe & PI0113110 & 07/Ago/2001 & 20/Mar/2018 & 20/Mar/2028 & 6,6 \\
\hline Daclatasvir & PI0716483 & 09/Ago/2007 & 09/Out/2018 & 09/Out/2028 & 1,2 \\
\hline Dasatinibe & PI0009721 & 12/Abr/2000 & 21/Nov/2018 & $21 /$ Nov/2028 & 8,6 \\
\hline Nilotinibe $* * *$ & PI0312464 & 04/Jul/2003 & 04/Jul/2017 & 04/Jul/2027 & 4,0 \\
\hline \multicolumn{6}{|l|}{$B$} \\
\hline Certolizumabe pegol & PI0106682 & 05/Jun/2001 & 18/Fev/2019 & 18/Fev/2029 & 7,7 \\
\hline Darunavir & PI0311176 & 16/Mai/2003 & 18/Fev/2019 & 18/Fev/2029 & 5,8 \\
\hline Sofosbuvir \# & PI0809654 & 26/Mar/2008 & 18/Fev/2019 & 18/Fev/2029 & 0,9 \\
\hline \multicolumn{6}{|l|}{ C } \\
\hline Adalimumabe \#\# & PI9707379 & 10/Fev/1997 & 23/Fev/2010 & $23 / \mathrm{Fev} / 2020$ & 3,0 \\
\hline Eculizumabe & PI9507594 & 01/Mai/1995 & 03/Nov/2009 & 03/Nov/2019 & 4,5 \\
\hline
\end{tabular}

\footnotetext{
* Considerando que as patentes do Grupo B foram concedidas em 18 de fevereiro de 2019;

** A data de extinção foi definida como o maior período entre 20 anos depois do depósito ou 10 anos da concessão;

*** Há um pedido dividido (PI0613605) que ainda não foi concedido;

\# O pedido (PI0410846) de 2004, já concedido após embates administrativos e judiciais, protege apenas um intermediário de síntese, e não a molécula. Foi selecionado o pedido que efetivamente impediria a competição, apesar de ter menor prazo potencial de extensão;

\#\# Dos dois pedidos depositados pelo titular no mesmo dia, foi selecionado o que terá maior extensão.

Fonte: elaboração própria.
} 
encontrados preços unitários do biossimilar adalimumabe e do genérico nilotinibe, para o mercado varejista, sendo, porém, superiores aos valores praticados nas compras do DLOG 24,25.

Na sétima etapa, a estimação dos custos potenciais do DLOG com a compra desses medicamentos considerou o VMU e a QMA nos respectivos períodos de extensão, comparando-os com preços unitários dos genéricos encontrados no mercado internacional, identificados na etapa seis. Foi utilizada a mesma QMA nos períodos de extensão, sem variação no VMU. Isso permitiu calcular uma alternativa efetiva para a compra desses medicamentos, caso não houvesse extensão de vigência.

$\mathrm{Na}$ oitava etapa, foram utilizados valores hipotéticos para as alternativas de compra do DLOG, a fim de englobar todos os medicamentos, posto que não foram encontrados genéricos e biossimilares para todos os casos. Portanto, para cada medicamento, foram estabelecidos três níveis hipotéticos de redução de preço: básico, médio e drástico. Para os medicamentos de base química, foram considerados genéricos 40\%, 60\% e 80\% mais baratos. Para os medicamentos biológicos, os biossimilares hipotéticos foram 10\%, 30\% e 50\% mais baratos.

Para os medicamentos químicos, a primeira estimação considera um genérico 40\% mais barato, porque o art. 12 da Resolução no 2 de 2004 da Câmara de Regulação do Mercado de Medicamentos (CMED) determina que o genérico deve ser, pelo menos, $35 \%$ mais barato que o medicamento de referência. Ademais, retirando os $5 \%$ de royalties cobrados em média, é possível afirmar que todo genérico é 40\% mais barato que o medicamento de referência, assim como consideram Hasenclever et al. 15 e Paranhos et al. 19. A segunda e a terceira reduções baseiam-se na informação da Pró-Genéricos (Associação Brasileira das Indústrias de Medicamentos Genéricos. Dados de mercado. http:// progenericos.org.br/dados-do-setor, acessado em 20/Abr/2019) de que os genéricos vendidos nas farmácias no Brasil são, em média, 60\% mais baratos e podem chegar a 80\% nos Estados Unidos. Para os medicamentos biológicos, é adotada a análise de Mulcahyet al. 26, de redução entre 10\% e 50\%, com média de cerca de $25 \%$.

\section{Apresentação e análise dos resultados}

Ao longo dos cinco anos analisados na pesquisa (2014 a 2018), o total dos gastos do DLOG com os nove medicamentos foi de R\$10,6 bilhões, com compras realizadas majoritariamente por meio de dispensa de licitação (77,8\%). Conforme Tabela 1, o Grupo A, com quatro medicamentos, foi responsável por R\$ 1,7 bilhão (16,2\%). O Grupo B, com três medicamentos, totalizou R\$ 2,8 bilhões (26\%). O Grupo C, com dois medicamentos, somou R\$ 6,1 bilhões (57,7\%). Não obstante todos os nove medicamentos gerarem gastos expressivos, é importante destacar os seguintes: o adalimumabe (35,7\%), indicado para artrite reumatoide, hidradenite supurativa, psoríase moderada a grave e uveíte não infecciosa; o eculizumabe (22\%), usado para hemoglobinúria paroxística noturna; e o sofosbuvir (16,3\%), para tratamento da hepatite C crônica. Esses medicamentos apresentam os maiores percentuais individuais de gastos entre os estudados.

Em termos de quantidade, é difícil comparar os medicamentos, posto que são somadas diferentes concentrações para alguns, e algumas terapias são contínuas. Dessa maneira, as informações de quantidade servem, principalmente, para o cálculo do VMU. Nesse indicador, o eculizumabe se destaca, tendo um VMU de R\$21.805,43, seguido de longe pelo golimumabe, com VMU de R\$ 1.472,35.

Para o Grupo C, a título de comparação sobre o efeito da extinção das patentes no preço, foram estimados os VMUs para a mesma quantidade de tempo antes e depois da extinção das patentes. Considerando um período de dois anos, o adalimumabe ficou 22,3\% mais barato após a extinção da patente. Considerando um período de 16 meses, o eculizumabe ficou 43,3\% mais caro. Dessa maneira, não foi possível afirmar que o preço do medicamento de referência cai sempre que a patente é extinta, já que a existência de concorrentes efetivos e potenciais terá sempre grande influência sobre a determinação de preço.

Reconhecendo o peso que esses medicamentos têm nos gastos do SUS e o planejamento do DLOG, é importante entender o impacto que a extensão produziria. Portanto, foi estimada a extensão de vigência para as patentes relacionadas conforme a Metodologia. Utilizando o GMA, também foi possível estimar o gasto total que o DLOG terá com a compra dos nove medicamentos em seus respectivos períodos de extensão. 
Tabela 1

Valores e quantidades de compra dos medicamentos pelo Departamento de Logística em Saúde do Ministério da Saúde (2014-2018).

\begin{tabular}{|c|c|c|c|c|c|}
\hline Grupo/Medicamento & $\begin{array}{l}\text { Gasto total } \\
\text { (R\$ milhão) }\end{array}$ & Quantidade total (mil) & VMU (R\$) & QMA (mil) & $\begin{array}{c}\text { GMA } \\
\text { (R\$ milhão) }\end{array}$ \\
\hline \multicolumn{6}{|l|}{ A } \\
\hline Golimumabe & 541,9 & 368 & $1.472,35$ & 74 & 108,4 \\
\hline Daclatasvir & 679,1 & 6.706 & 102,91 & 1.083 & 111,5 \\
\hline Dasatinibe & 307,4 & 3.940 & 99,52 & 335 & 33,4 \\
\hline Nilotinibe & 197,7 & 6.942 & 28,48 & 1.388 & 39,5 \\
\hline Total & $1.726,0$ & 17.955 & - & - & 292,8 \\
\hline \multicolumn{6}{|l|}{$B$} \\
\hline Certolizumabe pegol & 169,4 & 325 & 520,77 & 65 & 33,9 \\
\hline Darunavir & 867,0 & 110.503 & 7,89 & 15.947 & 125,8 \\
\hline Sofosbuvir & $1.732,3$ & 6.700 & 258,57 & 1.675 & 433,1 \\
\hline Total & $2.768,8$ & 117.528 & - & - & 592,8 \\
\hline Total A + B & $4.494,8$ & 135.483 & - & - & 885,6 \\
\hline \multicolumn{6}{|l|}{$\mathrm{C}$} \\
\hline Adalimumabe & $3.798,2$ & 4.320 & 713,79 & 864 & 616,6 \\
\hline Eculizumabe & $2.341,7$ & 102 & $21.805,43$ & 20 & 443,6 \\
\hline Total & $6.139,8$ & 4.421 & - & - & $1.060,2$ \\
\hline Total A + B + C & $10.634,6$ & 139.905 & - & - & $1.945,8$ \\
\hline
\end{tabular}

QMA: quantidade média anual; VMU: valor médio unitário.

Nota: quantidade medida em comprimido/seringa. O VMU e a QMA foram ponderados pelas diferentes concentrações do mesmo medicamento e pelos anos de incorporação ao Sistema Único de Saúde, posto que daclatasvir e sofosbuvir somente foram incorporados em 2015. Valores atualizados para janeiro de 2019.

Fonte: elaboração própria, com base nos dados do Painel de Compras do Governo Federal (http://paineldecompras. planejamento.gov.br/, acessado em 18/Fev/2019).

A estimativa de gasto com compra do Grupo A é de R $\$ 1,3$ bilhão (18,9\%) para todos os respectivos períodos de extensão. Para o Grupo B, esse valor é pouco maior: R\$1,4 bilhão (20,1\%). Novamente, o Grupo C lidera os custos, com R \$ 4,2 bilhões (61\%) de gastos estimados. Assim, o gasto total para o DLOG durante a extensão das patentes dos nove medicamentos foi calculado em $\mathrm{R} \$ 6,8$ bilhões, como visto na Tabela 2. Por conseguinte, é imperioso que sejam procuradas maneiras legais e constitucionais de reduzir esse gasto.

Para criar uma primeira perspectiva do quanto a extensão da vigência é responsável por esse gasto, foi calculada a economia que o DLOG teria se pudesse comprar os genéricos já disponíveis no mercado. Conforme indicado na Tabela 3, o genérico do darunavir encontrado é 58,1\% mais barato que o VMU praticado nas compras do DLOG, e o genérico do dasatinibe, 77,1\% mais barato. Não obstante serem reduções significativas, o daclatasvir e o sofosbuvir se destacam pela enorme diferença: seus genéricos são vendidos a valores $99,1 \%$ e $98,9 \%$ mais baratos, respectivamente. Se houvesse somente a troca desses quatro medicamentos de referência por seus genéricos, seria possível poupar praticamente $\mathrm{R} \$ 1,2$ bilhão $(75,5 \%)$ ao total do tempo de extensão.

Tendo em vista o potencial de redução de gastos com a compra de genéricos e biossimilares, foram estipulados três níveis de redução para os VMUs de cada medicamento. Primeiramente, a redução básica corresponderia à substituição dos medicamentos de referência por versões 10\% (biossimilares) e 40\% (genéricos) mais baratas, como apresentado na Tabela 4. Nesse cenário bastante conservador, o gasto do DLOG com os medicamentos do Grupo A poderia ser reduzido em R\$ 302,2 milhões; com os do Grupo B, R\$ 472,6 milhões; e, com os do Grupo C, R\$ 417,6 milhões. Portanto, uma redução básica já seria suficiente para o DLOG deixar de gastar R \$1,2 bilhão (17,4\% a menos), somando todos os períodos de extensão. Em termos de gasto anual, a economia seria de $\mathrm{R} \$ 417,6$ milhões (21,5\% a menos). Isto é, mesmo sendo conservadora, a redução já seria considerável. 
Tabela 2

Gasto total durante a extensão.

\begin{tabular}{|c|c|c|}
\hline Grupo/Medicamento & Extensão (anos) & $\begin{array}{l}\text { Gasto total durante a extensão } \\
\text { ( } R \$ \text { milhão) }\end{array}$ \\
\hline \multicolumn{3}{|l|}{$A$} \\
\hline Golimumabe & 6,6 & 717,6 \\
\hline Daclatasvir & 1,2 & 130,4 \\
\hline Dasatinibe & 8,6 & 287,5 \\
\hline Nilotinibe & 4,0 & 158,3 \\
\hline Total & - & $1.293,8$ \\
\hline \multicolumn{3}{|l|}{ B } \\
\hline Certolizumabe pegol & 7,7 & 261,3 \\
\hline Darunavir & 5,8 & 725,8 \\
\hline Sofosbuvir & 0,9 & 390,4 \\
\hline Total & - & $1.377,4$ \\
\hline Total A + B & - & $2.671,2$ \\
\hline \multicolumn{3}{|l|}{ C } \\
\hline Adalimumabe & 3,0 & $1.981,8$ \\
\hline Eculizumabe & 4,5 & $2.194,4$ \\
\hline Total & - & $4.176,2$ \\
\hline Total A + B + C & - & $6.847,5$ \\
\hline
\end{tabular}

Nota: o Grupo C tem a particularidade de englobar dois medicamentos que já estão no que seriam seus períodos de extensão, se esta for obtida pela via judicial. Portanto, parte do gasto apresentado é efetivo, já ocorrido até 2018, e parte é potencial, no período que restaria da extensão.

Fonte: elaboração própria, com base nos dados do Painel de Compras do Governo Federal (http://paineldecompras. planejamento.gov.br/, acessado em 18/Fev/2019).

\section{Tabela 3}

Custo potencial da extensão, considerando preços efetivos de genéricos.

\begin{tabular}{|c|c|c|c|c|}
\hline Grupo/Medicamento & $\begin{array}{l}\text { Gasto anual } \\
\text { (R\$ milhão) }\end{array}$ & $\begin{array}{l}\text { Gasto total durante a } \\
\text { extensão ( } R \$ \text { milhão) }\end{array}$ & $\begin{array}{c}\text { Redução de preço } \\
\text { efetiva (\%) }\end{array}$ & $\begin{array}{l}\text { Custo total potencial } \\
\text { (R\$ milhão) }\end{array}$ \\
\hline \multicolumn{5}{|l|}{ A } \\
\hline Daclatasvir & 111,5 & 130,4 & 99,1 & 129,3 \\
\hline Dasatinibe & 33,4 & 287,5 & 77,1 & 221,6 \\
\hline Total & 144,8 & 417,9 & - & 350,9 \\
\hline \multicolumn{5}{|l|}{$B$} \\
\hline Darunavir & 125,8 & 725,8 & 58,1 & 421,7 \\
\hline Sofosbuvir & 433,1 & 390,4 & 98,9 & 385,9 \\
\hline Total & 558,9 & $1.116,1$ & - & 807,7 \\
\hline Total A + B & 703,8 & $1.534,0$ & - & $1.158,5$ \\
\hline
\end{tabular}

Nota: taxa de câmbio do dólar de $\mathrm{R} \$ 3,73$ em 18 de fevereiro de 2019, com base no conversor de moedas do Banco Central do Brasil (https://www.bcb. gov.br/conversao, acessado em 29/Abr/2019).

Fonte: elaboração própria, com base nos dados da Unitaid 21, Organização Mundial da Saúde 22, Médicos Sem Fronteiras 23, Caprane (https://www. caprane.com/product/dasa-50-dasatinib-50mg/, acessado em 22/Abr/2019) e Painel de Compras do Governo Federal (http://paineldecompras. planejamento.gov.br/, acessado em 18/Fev/2019). 
Tabela 4

Custo potencial da extensão, considerando preços hipotéticos dos genéricos e biossimilares.

\begin{tabular}{lccc}
\hline Grupo/Medicamento & Redução básica * & $\begin{array}{c}\text { Custo da extensão } \\
\text { Redução média ** }\end{array}$ & Redução drástica *** \\
\hline A & & & \\
Golimumabe & 71,8 & 215,3 & 358,8 \\
Daclatasvir & 52,2 & 78,3 & 104,3 \\
Dasatinibe & 115,0 & 172,5 & 230,0 \\
$\quad$ Nilotinibe & 63,3 & 95,0 & 126,6 \\
$\quad$ Total & 302,2 & 561,0 & 819,7 \\
B $\quad$ & & 130,7 \\
$\quad$ Certolizumabe pegol & 26,1 & 78,4 & 580,6 \\
$\quad$ Darunavir & 290,3 & 435,5 & 312,3 \\
$\quad$ Sofosbuvir & 156,1 & 234,2 & $1.023,6$ \\
$\quad$ Total & 472,6 & 748,1 & $1.843,3$ \\
Total A + B & 774,8 & $1.309,0$ & \\
C & & & 990,9 \\
$\quad$ Adalimumabe & 198,2 & 594,5 & $1.097,2$ \\
Eculizumabe & 219,4 & 658,3 & $2.088,1$ \\
Total & 417,6 & $1.252,9$ & $3.931,4$ \\
Total A + B + C & $1.192,4$ & $2.561,9$ &
\end{tabular}

* Biossimilares $10 \%$ e genéricos $40 \%$ mais baratos;

** Biossimilares $30 \%$ e genéricos $60 \%$ mais baratos;

$* * *$ Biossimilares $50 \%$ e genéricos $80 \%$ mais baratos.

Fonte: elaboração própria, com base nos dados do Painel de Compras do Governo Federal (http://paineldecompras.

planejamento.gov.br/, acessado em 18/Fev/2019).

Em seguida, foi estimado o custo da extensão se os medicamentos fossem substituídos por genéricos e biossimilares com redução média: 30\% para os biológicos e 60\% para os de base química. Assim, os medicamentos do Grupo A poderiam ser R\$ 561 milhões mais baratos, os do Grupo B, R\$ 748,1 milhões, e os do Grupo C, R\$ 1,3 bilhão. Por conseguinte, dada uma redução de nível médio, a troca por esses genéricos e biossimilares hipotéticos evitaria o gasto total de R $\$ 2,6$ bilhões (37,4\% a menos) e, por ano, R\$ 806,7 milhões (41,5\% a menos) para o DLOG.

Por fim, foram considerados os maiores cenários hipotéticos de redução: $50 \%$ para os biossimilares e $80 \%$ para os medicamentos de base química. Dessa maneira, o horizonte hipotético de redução seria de R\$ 3,9 bilhões (57,4\% a menos), ao total, com uma economia anual de R\$ 1,2 bilhão (61,5\% a menos). Individualmente, o Grupo A ficaria R \$ 819,7 milhões mais barato, o Grupo B, R\$ 1 bilhão, e o Grupo C, R\$2,1 bilhões.

Dada a tese adotada neste artigo de que existe uma expectativa de extensão das patentes mailbox que afeta a concorrência de medicamentos protegidos por essas patentes, é possível calcular o custo efetivo adicional que o Grupo C já gerou ao DLOG, no tempo de extensão potencial desses pedidos, entre as datas em que suas patentes completaram vinte anos do depósito e a coleta de dados desta pesquisa, em 18 de fevereiro de 2019. Nesse período, foram gastos R\$ 3,1 bilhões com os dois medicamentos. Assim, o custo efetivo para o Grupo C de uma hipotética redução básica referente a esse período seria de R \$ 309,8 milhões. Se a redução fosse média, seriam R \$ 929,5 milhões. Se a redução fosse drástica, o DLOG teria economizado R\$ 1,5 bilhão, só com esses dois medicamentos nos períodos em que já foram comprados apenas os medicamentos de referência, apesar de não haver proteção patentária efetiva e de existir biossimilar pelo menos para o adalimumabe. Ao total dos períodos de extensão dos dois medicamentos, com garantia judicial, o custo global, considerando a redução drástica, subiria para $\mathrm{R} \$ 2,1$ bilhões. 


\section{Conclusões e implicações de políticas}

A pesquisa que embasa este artigo teve como objetivo realizar uma projeção dos custos adicionais para a compra de medicamentos pelo SUS da extensão de patentes farmacêuticas. Para isso, foram identificados os medicamentos com maiores gastos nas compras centralizadas do Ministério da Saúde que apresentassem situação patentária com extensão ou potencial de extensão. Os nove medicamentos selecionados foram divididos em três grupos, e os custos adicionais projetados devido à extensão foram calculados de duas maneiras. Primeiramente, consideraram-se os preços de genéricos no mercado internacional. Em seguida, levou-se em consideração a hipótese de genéricos 40\%, 60\% e 80\% mais baratos e biossimilares com preços $10 \%, 30 \%$ e $50 \%$ menores.

$\mathrm{O}$ custo de continuar comprando os quatro medicamentos de referência cujos genéricos já são vendidos internacionalmente foi estimado em $\mathrm{R} \$ 1,2$ bilhão. Essa estimativa é influenciada pelo fato de dois genéricos estrangeiros serem aproximadamente $99 \%$ mais baratos que o preço pago pelo DLOG na compra dos medicamentos de referência no Brasil. É impressionante que seja praticado no exterior preço tão mais baixo, haja vista o volume e o caráter planejado e centralizado das compras. O Brasil é o sexto maior mercado farmacêutico do mundo, com grande expressão do SUS 27 . Assim, deveria ter um maior poder de barganha na negociação dos preços de suas compras.

Por um lado, comprar os genéricos ou biossimilares que tivessem as reduções de preço hipotéticas mais básicas já reduziria em $\mathrm{R} \$ 1,2$ bilhão o custo total da compra dos nove medicamentos com extensão das patentes. Por outro lado, as maiores reduções de preço - que estão longe de serem impossíveis - evitariam R\$ 3,9 bilhões nos gastos do SUS. Como o gasto total para os respectivos períodos de extensão foi estimado em R \$ 6,8 bilhões, seria, portanto, uma redução de 57,4\%. Atualizando todos os valores para janeiro de 2019, a redução anual dos custos que o DLOG teria por comprar esses genéricos e biossimilares mais baratos seria de $\mathrm{R} \$ 1,2$ bilhão, que representa 1,1\% do orçamento do SUS de 2018 (Governo Federal. Portal da Transparência. http://www.portaltransparencia.gov.br, acessado em 22/Abr/2019), 5,4\% dos gastos com medicamentos pelos governos federal, estadual e municipal em 201613 e 57,7\% dos gastos da indústria farmacêutica em P\&D no Brasil em 2014 28. Colocando em perspectiva os valores encontrados, é reforçada a necessidade de atenção à regulação patentária de medicamentos no Brasil, dado que poucos medicamentos já podem gerar uma pressão fiscal significativa no orçamento do SUS e, consequentemente, no acesso a medicamentos pela população. Não há solução simples para esse problema, mas é possível identificar três encaminhamentos complementares que poderiam contribuir para sua solução: solicitação de exame prioritário, contestação judicial e restruturação administrativa.

O primeiro encaminhamento diz respeito a uma atuação mais proativa do Ministério da Saúde no pedido de exame prioritário para os medicamentos com pedidos de patentes com potencial de extensão de vigência - aqui identificadas três delas no Grupo B. Isso impediria que tais medicamentos retardassem a possibilidade de entrada de genéricos e de biossimilares no mercado e representassem maior ônus para o SUS. Esse procedimento foi permitido pelo INPI em 2013, por meio da Portaria no 80, mas ainda é muito pouco utilizado pelo Ministério 11,16.

O segundo encaminhamento seria pela declaração do Superior Tribunal Federal (STF) de inconstitucionalidade do parágrafo único do art. 40 da LPI, como proposto na Ação Direta de Inconstitucionalidade (ADI) no 5.529, de 2016. Isso extinguiria o mecanismo TRIPS-plus de mínimo de vigência, permitindo que os medicamentos genéricos e biossimilares sejam produzidos ao fim dos vinte anos de depósito dos pedidos, sem prejuízo da expectativa de direito durante o exame. Se isso não acontecer por meio de ADI, será pelo Recurso Extraordinário recebido em março de 2019, em que é pedido o mínimo de vigência das patentes mailbox - que a LPI expressamente nega (art. 229, parágrafo único). Se o STF não declarar todo o mínimo de vigência inconstitucional, pelo menos que mantenha a decisão do legislador na LPI ao expressamente rejeitar a extensão da vigência das patentes mailbox. Ao longo deste artigo, foi mostrado que apenas dois medicamentos protegidos por patentes mailbox podem gerar até R\$2,1 bilhões de custos adicionais ao DLOG.

Não obstante a relevância da discussão jurídica, existe uma realidade administrativa imperiosa. É inaceitável que o Brasil tenha um dos escritórios mais ineficientes em exame patentário, não pela falta de qualificação, mas pelo número insuficiente de examinadores 16. A razão backlog por examinador (641 em janeiro de 2019, segundo o INPI 17) provoca que o escritório, no geral, seja ineficiente, 
fazendo com que os pedidos de patentes farmacêuticas esperem por quase sete anos para que algum examinador consiga alcançá-los na pilha do backlog.

Por conseguinte, o terceiro encaminhamento seria a melhor estruturação administrativa do INPI, para garantir que ele tenha capacidade de exercer sua função de examinador técnico de patentes com qualidade, eficiência e independência. A contratação e treinamento de examinadores, assim como o fortalecimento da carreira de examinador de patente, são essenciais para o bom funcionamento do órgão. Ademais, não menos importante é a efetivação de sua autonomia financeira e administrativa, que foi prevista na lei de criação (Lei no 5.648/1970, art. 3o) e na LPI (art. 239), mas que ainda não é uma realidade. O INPI precisa de condições fáticas e práticas de melhorar seus processos de exame de patentes, podendo reduzir o tempo médio de análise. Assim, o parágrafo único do art. 40 deixará de ser a regra - 92,2\% das patentes farmacêuticas que podem ter extensão de fato o recebem ${ }^{11}$ - e passará a ser exceção que o legislador originalmente desenhou.

\section{Colaboradores}

J. Paranhos participou da concepção e projeto, análise e interpretação dos dados, redação e revisão crítica relevante do conteúdo intelectual do artigo e aprovação final da versão a ser publicada. E. Mercadante e L. Hasenclever colaboraram na concepção e projeto, análise e interpretação dos dados, redação e revisão crítica relevante do conteúdo intelectual do artigo.

\section{Informações adicionais}

ORCID: Julia Paranhos (0000-0003-4425-7484); Eduardo Mercadante (0000-0001-5597-4863); Lia Hasenclever (0000-0003-1384-6323).

\section{Agradecimentos}

Os autores agradecem ao professor da Faculdade de Farmácia da Universidade Federal do Rio de Janeiro, Thiago Azeredo, pela colaboração na busca de preços internacionais, e às especialistas em patentes colaboradoras da empresa Libbs Farmacêutica, pela busca das patentes referentes aos medicamentos do estudo. O projeto de pesquisa foi financiado pela empresa Libbs Farmacêutica.

\section{Conflito de Interesses}

A pesquisa foi financiada pela empresa Libbs Farmacêutica, que não teve qualquer influência na análise e publicação dos resultados.

\section{Referências}

1. Pavitt K. Sectoral patterns of technical change: towards a taxonomy and a theory. Res Policy 1984; 13:343-73.

2. Mansfield E. Patents and innovation: an empirical study. Manage Sci 1986; 32:173-81.

3. Levin R, Klevorick A, Nelson R, Winter S, Gilbert R, Griliches Z. Appropriating the returns from industrial research and development. Brookings Pap Econ Act 1987; (3):783-831.

4. Cohen W, Goto A, Akiya A, Nelson R, Walsh J. $\mathrm{R} \& \mathrm{D}$ information flows and patenting in Japan and the United States. In: Granstrand O, editor. Economics, law and intellectual property. Cheltenham: Edward Elgar; 2003. p. 123-54.

5. Granstrand O. Innovation and intellectual property rights. In: Fagerberg J, Mowery D, Nelson R, editors. The Oxford handbook of innovation. New York: Oxford University Press; 2005. p. 266-90.

6. Andersen B. If 'intellectual property rights' is the answer, what is the question? Revising the patent controversies. In: Andersen B, editor. Intellectual property rights: innovation, governance and the institutional environment. Cheltenham: Edward Elgar Publishing; 2006. p. 109-47.

7. Rockett K. Property rights and invention. In: Hall B, Rosenberg N, editors. Economics of innovation. v. 1. New York: Elsevier; 2010. p. 315-80.

8. Bermudez J, Epsztejn R, Oliveira M, Hasenclever L. O Acordo TRIPS da OMC e a proteção patentária no Brasil: mudanças recentes e implicações para a produção local e o acesso aos medicamentos. Rio de Janeiro: Escola Nacional de Saúde Pública, Fundação Oswaldo Cruz; 2000. 
9. Chaves G, Oliveira M, Hasenclever M, Melo L. A evolução do sistema internacional de propriedade intelectual: proteção patentária para o setor farmacêutico e acesso a medicamentos. Cad Saúde Pública 2007; 23:257-67.

10. Correa C. Mitigating the regulatory constraints imposed by intellectual property rules under free trade agreements. Geneva: South Center; 2017. (South Center Reasearch Paper, 74).

11. Mercadante E. Concessão de patentes farmacêuticas no Brasil pós-Acordo TRIPS [Dissertação de Mestrado]. Rio de Janeiro: Instituto de Economia, Universidade Federal do Rio de Janeiro; 2019.

12. Magalhães M, Beghin N, David G. Brasil: recursos federais destinados à assistência farmacêutica em tempos de austeridade. Avaliação das execuções financeiras de 2015 a 2017. Brasília: Instituto de Estudos Socioeconômicos; 2018.

13. Pimentel V. Parcerias para o desenvolvimento produtivo de medicamentos no Brasil sob a ótica das compras públicas para inovação: 2009-2017 [Dissertação de Mestrado]. Rio de Janeiro: Instituto de Economia, Universidade Federal do Rio de Janeiro; 2018.

14. Brasil. Lei no 9.279 , de 14 de maio de 1996 . Regula direitos e obrigações relativos à propriedade industrial. Diário Oficial da União 1996; 15 mai.

15. Hasenclever L, Lopes R, Chaves G, Vieira M. O Instituto de Patentes Pipeline e o acesso a medicamentos: aspectos econômicos e jurídicos deletérios à economia da saúde. Rev Direito Sanit 2010; 11:164-88.

16. Abreu J. Prospecção tecnológica aplicada na otimização da concessão de patentes no Brasil: estudo de caso em patentes de medicamentos imunossupressores [Tese de Doutorado]. Rio de Janeiro: Instituto de Economia, Universidade Federal do Rio de Janeiro; 2017.

17. Instituto Nacional da Propriedade Industrial. Relatório estoque de pedidos pendentes: janeiro 2019. Brasília: Instituto Nacional da Propriedade Industrial; 2019.

18. Instituto Nacional da Propriedade Industrial. Relatório de atividades 2018. Brasília: Instituto Nacional da Propriedade Industrial; 2018.
19. Paranhos J, Hasenclever L, Chaves G, Cunha G. Extensão das patentes e custos para o SUS. Relatório de pesquisa. Rio de Janeiro: Associação Brasileira Interdisciplinar de AIDSBrasília: Instituto Nacional da Propriedade Industrial; 2016.

20. Jannuzzi AHL, Vasconcellos AG. Quanto custa o atraso na concessão de patentes de medicamentos para a saúde no Brasil? Cad Saúde Pública 2017; 33:e00206516.

21. Unitaid. Technology and market landscape hepatitis C medicines. Geneva: World Health Organization; 2017.

22. World Health Organization. Regional workshop on scaling up health sector response to viral hepatitis in South-East Asia Region. http://origin.searo.who.int/entity/hepatitis/ vhr_29aug17.pdf (acessado em 22/Abr/2019).

23. Médecins Sans Frontières. Stop senseless deaths. Paris: Médecins Sans Frontières; 2018. (Technical Breafing Document).

24. National Institute for Health and Care Excellence. Dasatinib, nilotinib and high-dose imatinib for treating imatinib-resistant or intolerant chronic myeloid leukaemia. https://www. nice.org.uk/guidance/ta425 (acessado em 22/ Abr/2019).

25. Ghangurde A. Here's what competition looks like in India's Humira biosimilars market. https://pharmaintelligence.informa.com/re sources/product-content/heres-what-compe tition-looks-like-in-indias-humira-biosimi lars-market (acessado em 22/Abr/2019).

26. Mulcahy A, Hlavka J, Case S. Biosimilar cost savings in the United States. Rand Health Q 2018; 7:3.

27. Associação da Indústria Farmacêutica de Pesquisa. Guia Interfarma 2018. https://www. interfarma.org.br/public/files/biblioteca/guia -interfarma-2018-interfarma.pdf www.inter farma.org.br/guia/guia-2017/dados-do-setor/ (acessado em 24/Abr/2019).

28. Instituto Brasileiro de Geografia e Estatística. Pesquisa de Inovação 2014. Rio de Janeiro: Instituto Brasileiro de Geografia e Estatística; 2016. 


\section{Abstract}

The sole paragraph of Article 40 of the Brazilian Industrial Property Law determines the extension of the patent term in case of delayed prosecution, guaranteeing 10 years of protection from grant. Originally conceived as an exception, this provision became the rule in the case of drug patents, prolonging period of exclusive exploitation. This situation impacts both the out-of-pocket spending by families and the budget of the Brazilian Unified National Health System (SUS). This study explores the cost to SUS of extending the patent term for nine drugs, which delays the launch of generic or biosimilar alternatives. Three groups were analyzed: (a) four patents already extended; (b) three that will be extended if granted; and (c) two drugs with mailbox patents that are in the public domain, but that may have the extension granted by court order. Based on the unit values and the total amounts of purchases by the Logistics Department of the Ministry of Health (DLOG), a total expenditure of BRL 10.6 billion on the nine drugs from 2014 to 2018 was estimated. The total projected expenditure for the respective extension periods was thus BRL 6.8 billion. A market price search for generic alternatives yielded potential savings of BRL 1.2 billion in expenditures by DLOG on four drugs alone. Finally, based on the minimum, average, and maximum market price reductions, potential savings of up to BRL 3.9 billion were estimated for DLOG on the purchase of the nine medicines, if the patent terms were not extended.

Patent; Drug Price; Costs and Cost Analysis; Unified Health System

\section{Resumen}

El párrafo único del art. 40 de la Ley de Propiedad Industrial brasileña establece la extensión de la vigencia de patentes, debido al retraso de su examen, garantizando al menos 10 años a partir de su concesión. Concebido como excepcional, este recurso se convirtió en una regla en el caso de las patentes farmacéuticas, prolongando el plazo de exclusividad de explotación de medicamentos. Esto impacta en el consumo privado y el presupuesto del Sistema Único de Salud de Brasil (SUS). Este trabajo investiga el coste para el SUS de la extensión de vigencia de nueve medicamentos, por estar prohibida la compra de versiones genéricas o biosimilares. Se analizaron tres grupos: (a) cuatro ya en extensión; (b) tres que, si fueran concedidas las patentes, tendrán extensión; y (c) dos con patentes mailbox que son de dominio público, pero pueden contar con una extensión garantizada judicialmente. A partir de valores unitarios, y cantidades totales de compra por parte del Departamento de Logística en Salud del Ministerio de la Salud (DLOG), se estimó un gasto total de BRL 10,6 billones con los nueve medicamentos de 2014 a 2018. De este modo, se proyectó el gasto total en los respectivos periodos de extensión: $B R L$ 6,8 billones. Una búsqueda de mercado con precios de alternativas genéricas arrojó una reducción potencial de BRL 1,2 billón para el gasto del DLOG, con solo cuatro medicamentos. Finalmente, basándose en las reducciones mínimas, medias y máximas del mercado, se encontró un ahorro potencial de hasta BRL 3,9 billones en el gasto del DLOG con la compra de los nueve medicamentos, si no existiera un recurso de extensión de la vigencia.

Patente; Precio de Medicamento; Costos y Análisis de Costo; Sistema Único de Salud

Recebido em 02/Set/2019

Versão final reapresentada em 16/Mar/2020

Aprovado em 27/Abr/2020 